\title{
VIABILIDAD DE UTILIZAR CENIZAS DE CALDERAS INDUSTRIALES EN LA DOSIFICACIÓN DE HORMIGÓN ESTRUCTURAL
}

\section{ARTÍCULO ORIGINAL}

JUSTINO, Lucas Diego de Souza ${ }^{1}$

JUSTINO, Lucas Diego de Souza. Viabilidad de utilizar cenizas de calderas industriales en la dosificación de hormigón estructural. Revista Científica Multidisciplinar Núcleo do Conhecimento. Año 06, Ed. 09, Vol. 02, págs. 81-97. Septiembre 2021. ISSN: 2448-0959, Enlace de acceso: https://www.nucleodoconhecimento.com.br/ingenieria-civil/hormigon-estructural, DOI: https://www.nucleodoconhecimento.com.br/ingenieria-civil/hormigon-estructural

\section{RESUMEN}

La industrialización y el crecimiento acelerado de la población generan efectos secundarios en diversos aspectos sociales, y el tema ambiental es preocupante debido a los impactos causados por la evolución social. La gestión de los residuos industriales es un gran reto que implica tanto el control de su generación como la correcta eliminación, garantizando la sostenibilidad medioambiental. Los residuos de cenizas de calderas se encuentran en abundancia en las fábricas que utilizan este equipo para la generación de vapor. Esta abundancia se produce debido a la falta de un lugar para la eliminación o reutilización adecuada del residuo. Ante este escenario, este artículo tenía como guía la pregunta: ¿Sería posible utilizar este residuo en la producción de hormigón estructural? El objetivo de este estudio fue clasificar el residuo definiendo su posible forma de uso en la dosificación de hormigón y realizar dosificaciones experimentales con el uso de cenizas de calderas industriales para evaluar sus posibles contribuciones técnicas a las propiedades básicas del hormigón. Para ello, las muestras fueron recolectadas por una industria

${ }^{1}$ Graduado en Ingeniería Civil. ORCID: 0000-0003-2630-8866

RC: 97179

Disponible: https://www.nucleodoconhecimento.com.br/ingenieria-civil/hormigonestructural 
instalada en la ciudad de Uberlândia, Estado de Minas Gerais, activa en varios sectores como la agricultura, la nutrición animal, la farmacéutica y otros. La clasificación de las cenizas de caldera se realizó aplicando los procedimientos normativos brasileños y los parámetros utilizados para la clasificación de aglutinantes y agregados para concreto. Teniendo en cuenta su curva granulométrica y densidad, el residuo se clasificó como agregado ligero y muy delgado, adoptando así la metodología de reemplazo parcial del agregado delgado por cenizas de caldera. Se verificó que había una reducción en la trabajabilidad del hormigón en estado fresco proporcionalmente al contenido de residuos utilizado. Por lo tanto, es necesario utilizar aditivos superplastificantes en estos casos para mantener la trabajabilidad esperada. Se observó una reducción en la densidad del hormigón cuando se utilizó el residuo, considerando como necesaria la atención a esta propiedad del hormigón en relación con el contenido utilizado del residuo en la dosificación. También se observó al comparar las dosis con el uso del residuo en la dosis estándar, que hubo una reducción en la resistencia a la compresión. Sin embargo, no hubo una gran variación en la resistencia a la compresión entre las dosis con diferentes niveles de sustitución utilizados. Se concluyó, por tanto, considerando los niveles utilizados en este estudio, como factible el uso de este residuo en la producción de hormigón estructural.

Palabras clave: Hormigón, Biomasa, Ceniza de Caldera Industrial.

\section{INTRODUCCIÓN}

La Revolución Industrial comenzó en Inglaterra en el siglo 18 a partir de la creación de la máquina de vapor por el ingeniero escocés James Watt, impulsó la industrialización británica con el fin de transformar el sistema de producción. Este período según Cavalcante (2011) "fue el precursor del capitalismo, es decir, la transición del capitalismo comercial al capitalismo industrial". Esto significa que, en este contexto, el régimen de fabricación, antes utilizado, es sustituido por el régimen de facturas maquino, dando como resultado procesos productivos mucho más ágiles

$\mathrm{RC}: 97179$

Disponible: https://www.nucleodoconhecimento.com.br/ingenieria-civil/hormigonestructural 
capaces de poner en práctica la producción en masa, asegurando una mayor oferta de producto y bajos costes de producción, en comparación con el escenario anterior.

Las transformaciones que ocurrieron durante la Revolución Industrial se expandieron globalmente y acompañaron la evolución de la sociedad a lo largo del tiempo, modernizando y mejorando las prácticas de producción a lo largo de los años. Sin embargo, con el avance del proceso de industrialización, el crecimiento de la población mundial y en consecuencia la alta demanda de productos, el consumo de recursos naturales crece proporcionalmente a esta demanda, ya que estos recursos sirven como materia prima para los procesos de fabricación.

El proceso de crecimiento acelerado genera, por lo tanto, como efectos secundarios, los impactos ambientales causados por la explotación de los recursos naturales, sin una gestión adecuada centrada en el mantenimiento y conservación del medio ambiente. "La explotación de los recursos naturales se ha vuelto depredadora en favor de la obtención de capital" (GANZALA, 2018).

Los efectos negativos de la industrialización a lo largo de los siglos 20 y 21 relacionados con el medio ambiente no solo están vinculados al consumo insostenible de recursos naturales involucrados en los procesos de producción, sino también a la contaminación del agua, el suelo y el aire causada por desechos o desechos generados por procesos industriales. Esta realidad se observa tanto en los países desarrollados como en los países en desarrollo.

Varios procesos industriales utilizan equipos llamados calderas de generación de vapor en el proceso de producción de diversas industrias de azúcar y alcohol, fábricas de secado y hervido de granos, refrigeradores, industria metalúrgica, textil y en el sector de generación de energía. El tipo de caldera más común utilizado actualmente es la llamada caldera de biomasa. Estas calderas utilizan como material de combustión las diferentes formas de biomasa como: pino, eucalipto, cáscara de arroz y bagaza de caña de azúcar. Después de quemar la biomasa, las cenizas de la

RC: 97179

Disponible: https://www.nucleodoconhecimento.com.br/ingenieria-civil/hormigonestructural 
caldera se generan como residuos, por lo que la ceniza de la caldera consiste en la parte resistente a la quemadura, presente en la composición de la biomasa utilizada como combustible en las calderas industriales.

Las industrias que utilizan las calderas, apuntando a la eficiencia energética y al factor económico, aplican controles de procesos de quema de biomasa, que reducen al máxima la generación de este tipo de residuos. Sin embargo, la generación de residuos es inevitable en este proceso. Por lo tanto, la disponibilidad de este residuo se vuelve abundante. En estas industrias se acumulan grandes depósitos de este material debido a la falta de alternativas para el destino o reutilización del mismo. Estos depósitos ocupan grandes espacios físicos en las plantas de producción, que podrían ser mejor utilizados, considerando la actividad final de la industria en cuestión.

En el escenario encontrado de alta disponibilidad del residuo gris de la caldera y la falta de un sitio o actividad de eliminación adecuada para la reutilización del residuo, surge la pregunta fundamental para el desarrollo de este estudio: ¿sería posible utilizar este residuo en la producción de hormigón estructural? Teniendo en cuenta el ritmo acelerado del sector de la construcción y su búsqueda de innovaciones, los residuos generados podrían ser reutilizados en este segmento como una alternativa sostenible, con la posibilidad de promover beneficios técnicos y económicos tanto para la industria generadora de residuos como para las empresas productoras de hormigón.

La contribución ambiental es el principal y más significativo logro con el uso de residuos de cenizas de calderas industriales en la producción de hormigón estructural, pues habría una doble contribución ambiental para evitar la eliminación de residuos en el medio ambiente, y, de verificarse la posibilidad de su aplicación como reposición parcial de áridos naturales, se evitaría la extracción de parte de estos recursos naturales, que cada vez son más escasos.

RC: 97179

Disponible: https://www.nucleodoconhecimento.com.br/ingenieria-civil/hormigonestructural 
Las empresas productoras de hormigón tienen como característica el uso de materias primas, es decir, sin necesidad de grandes procesos de procesamiento, como arenas naturales, que se extraen de ríos o depósitos, y requieren un procesamiento simple como el tamizado para la eliminación de impurezas y la clasificación del tamaño de partícula. Por lo tanto, el uso de residuos en la producción de hormigón tiene una contribución económica tanto para la industria generadora de residuos, que proporciona los residuos sin necesidad de grandes procesos de procesamiento, como para las empresas productoras de hormigón, que se beneficiarían de la alta disponibilidad de residuos y su bajo costo, siendo utilizados como materia prima en su producción.

El objetivo general de este estudio es realizar la clasificación preliminar del residuo de ceniza de caldera industrial, definir su posible forma de uso en la dosificación de hormigón, realizar dosificaciones experimentales para verificar el rendimiento de las propiedades básicas del hormigón dosificado con el uso de ceniza de silla, evaluar posibles aportaciones técnicas y, por último, concluir la viabilidad del uso de este residuo para dosificación de hormigón estructural.

\section{METODOLOGÍA}

La concepción inicial de la posibilidad de hacer factible el uso de cenizas de caldera para la producción de hormigón surge de la idea de que, originalmente, solo se utilizaban tres materiales básicos en la producción de hormigón: cemento, agregado y agua. Sin embargo, para un mejor rendimiento tanto en el estado fresco como en el endurecido, se inició el uso de aditivos químicos. Después de algún tiempo, el uso de materiales cementicios de naturaleza orgánica se introdujo en este contexto como adiciones a la mezcla de concreto como escoria granulada, puzolanas, humo de sílice y cenizas volantes.

Las razones iniciales para utilizar estos materiales solían ser económicas: costaban menos que el cemento Portland, a menudo porque existían en depósitos naturales, requiriendo poco o ningún

RC: 97179

Disponible: https://www.nucleodoconhecimento.com.br/ingenieria-civil/hormigonestructural 
procesamiento, otras veces porque eran subproductos o rechazos de procesos industriales (NEVILLE, 1997, p. 81).

"La ceniza volante es el resultado de calderas en el proceso de quema de carbón en las que parte de la materia mineral se agrupa formando cenizas de rejilla, pero la mayor parte es arrastrada por la corriente de escape del gas, que se llama ceniza volante" (MEHTA; MONTEIRO, 1994).

Este estudio se realizó en abril de 2021, a través de la investigación de innovaciones para el segmento de producción de concreto en la región de Uberlândia, estado de Minas Gerais, donde se observó una gran cantidad de industrias instaladas en esta localidad, que utilizaban calderas como medio para generar vapor en sus procesos de producción y, por lo tanto, había una gran disponibilidad de cenizas de caldera, así como la gran necesidad de una adecuada eliminación ambiental o reutilización de los residuos.

\subsection{MUESTRA Y CARACTERIZACIÓN DE CENIZAS}

La muestra del material utilizado en este estudio fue proporcionada por una gran industria instalada en el municipio de Uberlândia, Estado de Minas Gerais, que opera en varios sectores como la agricultura, la nutrición animal, la farmacéutica y otros. Las cenizas resultantes del proceso de quema de biomasa de eucalipto se mezclan con agua para evitar la dispersión atmosférica, y se realizan en el proceso de decantación, donde se recogió la muestra. Por lo tanto, el material recogido presenta un alto contenido de humedad, verificado visualmente por el agua libre presente superficialmente en la muestra recogida, además del agua absorbida por el propio material.

Tras la recogida de la muestra y su conducción al laboratorio de materiales, se llevó a cabo el proceso de secado del material para iniciar su caracterización básica. Debido al alto contenido de humedad observado en la muestra, fue necesario iniciar el proceso de secado esparciendo el material exterior sobre un revestimiento

RC: 97179

Disponible: https://www.nucleodoconhecimento.com.br/ingenieria-civil/hormigonestructural 
plástico, evitando la contaminación y dispersiones del mismo. Después del secado parcial al aire libre, el material se llevaba al invernadero para finalizar el proceso de secado.

El índice de finura se determinó por el tamiz número 200, según NBR 11579 ABNT (2012). Durante la realización de esta prueba, se observó que en el material retenido en el tamiz número 200, la mayoría de ellos consistían en material fino, pero se observó la presencia de partículas con dimensiones de tamaño de partícula más grandes, pero en una pequeña proporción. El índice de finura encontrado para el material fue del $46 \%$. Este índice se consideró alto, en comparación con el cemento Portland, que tiene este índice máximo del 10\%, establecido por NBR 16697 (ABNT, 2018).

La masa específica aparente se determinó de acuerdo con NM 52 ABNT (2009). La ceniza presentaba una masa específica aparente de $0,26 \mathrm{~g} / \mathrm{cm}^{3}$. Para Bauer (2008), los agregados se pueden clasificar de acuerdo con su peso específico. Por lo tanto, la ceniza se clasificó, en este aspecto, como agregado ligero, y el peso específico encontrado fue equivalente al peso específico de agregados ligeros conocido, como ejemplo de agregado ligero a vermicusita, que tiene un peso específico de 0,3 g / $\mathrm{cm}^{3}$.

La caracterización granulométrica se realizó según NBR NM 248 ABNT (2003). La ceniza presentó distribución granulométrica según Tabla 1, módulo de finura de 0,74 y dimensión máxima de 1,2 mm.

Tabla 1 - Distribución granulométrica gris caldera

\begin{tabular}{|l|l|l|}
\hline $\begin{array}{l}\text { Tamiz } \\
\text { (mm) }\end{array}$ & Retenido (\%) & Acumulado (\%) \\
\hline $\mathbf{2 , 4}$ & 0,80 & 0,80 \\
\hline $\mathbf{1 , 2}$ & 2,47 & 3,27 \\
\hline
\end{tabular}

RC: 97179

Disponible: https://www.nucleodoconhecimento.com.br/ingenieria-civil/hormigonestructural 


\begin{tabular}{|l|l|l|}
\hline $\mathbf{0 , 6}$ & 5,74 & 9,01 \\
\hline $\mathbf{0 , 3}$ & 12,23 & 21,24 \\
\hline $\mathbf{0 , 1 5}$ & 18,43 & 39,67 \\
\hline Fondo & 60,33 & $* * *$ \\
\hline
\end{tabular}

Fuente: Autor (2021).

En base a las características básicas verificadas, se intentó asignar la clasificación inicial de la ceniza para definir su forma de aplicación en dosis concretas. Inicialmente se consideraron dos posibilidades que se describen a continuación.

La primera posibilidad de utilizar ceniza sería incorporarla a la dosificación del hormigón como adición, comenzando como referencia las adiciones de materiales como ejemplo de sílice activa, ampliamente utilizada en la producción de hormigón de alto rendimiento y para potenciar propiedades específicas del hormigón, como resistencias mecánicas, principalmente. Sin embargo, debido al perfil verificado de la ceniza en la determinación de su índice de finura y distribución granulométrica, se descartó esta posibilidad debido a la incompatibilidad de sus características con este tipo de adición.

Como segunda posibilidad de utilizar ceniza, se verificó su posible introducción como reemplazo de agregados en la dosificación de concreto. Se observaron los resultados obtenidos en relación al peso específico y, como ya se informó, se enmarcó como un agregado ligero. Otra característica analizada, considerando esta posibilidad de uso fue la clasificación de la ceniza en relación al rango granulométrico en el que se enmarcó, con base en la Tabla 2.

Cuadro 2. Clasificación de las arenas por rangos granulométricos

\section{Porcentajes} retenidos

RC: 97179

Disponible: https://www.nucleodoconhecimento.com.br/ingenieria-civil/hormigonestructural 


\begin{tabular}{|c|c|c|c|c|}
\hline Tamices (mm) & $\begin{array}{l}\text { Pista } 1 \text { - Muy } \\
\text { delgada }\end{array}$ & $\begin{array}{l}\text { Pista } 2 \\
\text { Delgado }\end{array}$ & $\begin{array}{l}\text { Pista } 3 \\
\text { Media }\end{array}$ & $\begin{array}{l}\text { Pista } 4 \\
\text { Grueso }\end{array}$ \\
\hline 6,3 & De 0 a 3 & De 0 a 7 & De 0 a 7 & De 0 a 7 \\
\hline 4,8 & De 0 a 5 & De 0 a 10 & De 0 a 11 & De 0 a 12 \\
\hline 2,4 & De 0 a 5 & De 0 a 15 & De 0 a 25 & De 5 a 40 \\
\hline 1,2 & De 0 a 10 & De 0 a 25 & De 10 a 45 & De 30 a 70 \\
\hline 0,6 & De 0 a 20 & De 21 a 40 & De 41 a 65 & De 66 a 85 \\
\hline 0,3 & De 50 a 85 & De 60 a 88 & De 70 a 92 & De 80 a 95 \\
\hline 0,15 & De 85 a 100 & De 90 a 100 & 90 a100 & 90 a 1 \\
\hline
\end{tabular}

Fuente: Bauer (2008).

La distribución granulométrica de las cenizas presentadas en la Tabla 1 no se enmarcó completamente en ninguno de los rangos de clasificación descritos en la Tabla 2. Sin embargo, se observaron características similares a la distribución clasificada como pista 1 - Muy delgada, enjustándose en este rango los porcentajes retenidos en los tamices con apertura de 2,4 mm, 1,2 mm y 0,6 $\mathrm{mm}$. En los demás tamices, los porcentajes retenidos se mantuvieron por debajo de los especificados en el cuadro 2.Se observó una alta concentración de material en la parte inferior de la serie de tamiz, es decir, material que pasa en el tamiz con una abertura de 0,15 $\mathrm{mm}$.

Se verificó, por tanto, que la ceniza presentaba una distribución granulométrica muy cercana a la banda 1 - Muy delgada, pero con un porcentaje significativo de material más delgado de lo previsto en este rango granulométrico, es decir, tendiendo a un rango anterior, si lo hubiera.

El módulo de finura de 0,74 , obtenido por la caracterización granulométrica de la ceniza, como ya se ha informado, reforzó la clasificación de la ceniza en un rango anterior a la banda 1 - Muy delgada, ya que el módulo de finura mínima para la clasificación en este rango sería 1,35 (BAUER, 2008).

RC: 97179

Disponible: https://www.nucleodoconhecimento.com.br/ingenieria-civil/hormigonestructural 
Debido a la incompatibilidad de la ceniza con el índice de finura de las adiciones utilizadas tradicionalmente y su clasificación parcial como un agregado muy delgado, se optó, por lo tanto, por estudiar la posibilidad de reemplazar agregados muy delgados con cenizas en la dosificación de concreto.

\subsection{NIVELES ESTÁNDAR DE CARRERA Y REEMPLAZO}

La definición del rasgo a utilizar como estándar se basó en las características de la ceniza, previamente determinadas. El trazo por defecto debe contener en su composición el agregado clasificado como muy delgado, que sería reemplazado por gris.

El aumento en el consumo de agua en la dosificación debido a la adición de cenizas fue un punto de atención en la definición del trazo estándar, ya que teniendo en cuenta la advertencia de Neville (1997) "la ceniza de paja de arroz tiene formas complejas, según la planta de origen y, por lo tanto, requiere mucha agua.", se observó el tiempo requerido para secar la muestra de ceniza de la caldera y se consideró que debido a su origen y superficie específica, posiblemente la adición de cenizas aumentaría el consumo de agua a la dosis en relación con la dosis estándar.

Para combatir el aumento en el consumo de agua en dosis con la adición de cenizas, Neville (1997) recomienda utilizar aditivos superplastificantes para lograr la trabajabilidad esperada. Por lo tanto, elegimos definir el rasgo estándar con el uso de este tipo de aditivo.

La carrera estándar se adoptó con la resistencia a la compresión requerida de 25 MPa y la dispersión entre 600 y 650 mm, determinada de acuerdo con NBR 15823-2 ABNT (2017). Se encontraron las siguientes proporciones para el rasgo de masa unitaria: $1: 0.59: 2.21: 1.11: 2.60$, con una relación a/c igual a 0.58 , se obtuvo el cuantitativo para la mezcla según la Tabla 3.

RC: 97179

Disponible: https://www.nucleodoconhecimento.com.br/ingenieria-civil/hormigonestructural 
Tabla 3 - Carrera estándar 25.0 MPa Dispersión 600 a 650 mm

\begin{tabular}{|l|l|l|}
\hline Cemento & $\mathbf{1 0}$ & $\mathbf{K g}$ \\
\hline Arena muy fina & 5,88 & $\mathrm{Kg}$ \\
\hline Arena media & 22,10 & $\mathrm{Kg}$ \\
\hline Grava 0 & 11,12 & $\mathrm{Kg}$ \\
\hline Grava 1 & 25,96 & $\mathrm{Kg}$ \\
\hline Aditivo plastisitante & 0,035 & $\mathrm{~L}$ \\
\hline $\begin{array}{l}\text { Aditivo } \\
\text { superplastificante }\end{array}$ & 0,10 & $\mathrm{~L}$ \\
\hline Agua & 5,8 & $\mathrm{~L}$ \\
\hline
\end{tabular}

Fuente: Autor (2021).

El agregado nombrado como "arena muy fina" en la Tabla 3 fue el agregado adoptado como reemplazable por cenizas de caldera. El agregado presentado en su distribución granulométrica, módulo de finura de 1.42, clasificándose en el rango granulométrico 1, que comprende agregados con módulo de finura entre 1.35 y 2.25. Por lo tanto, se clasificó como agregado muy delgado (BAUER, 2008).

La sustitución del árido muy fino por cenizas se realizó parcialmente, es decir, adoptando niveles de sustitución aleatorios y progresivos, con el fin de provocar la observación de las posibles influencias causadas por la sustitución del árido por cenizas de caldera. Como punto de partida, se adoptaron niveles de sustitución del $10 \%, 20 \%$ y $30 \%$ sobre la masa agregada muy fina presente en la dosis estándar del rasgo ya presentada en la Tabla 3.

La Tabla 4 muestra los rasgos unitarios de masa adoptados para cada contenido de sustitución establecido.

RC: 97179

Disponible: https://www.nucleodoconhecimento.com.br/ingenieria-civil/hormigonestructural 
Cuadro 4 - Trazas de unidades de masa adoptadas según el contenido de sustitución del agregado muy fino por cenizas de caldera

\begin{tabular}{|l|l|}
\hline $\begin{array}{l}\text { Contenido agregado de reemplazo de } \\
\text { cenizas }\end{array}$ & Rasgo de la unidad de masa \\
\hline $\mathbf{1 0} \%$ & $1: 0,06: 0,53: 2,21: 1,11: 2,60 \mathrm{a} / \mathrm{c}=$ \\
& 0,58 \\
\hline $\mathbf{2 0} \%$ & $1: 0,12: 0,47: 2,21: 1,11: 2,60 \mathrm{a} / \mathrm{c}=$ \\
& 0,58 \\
\hline $\mathbf{3 0} \%$ & $1: 0,18: 0,41: 2,21: 1,11: 2,60 \mathrm{a} / \mathrm{c}=$ \\
& 0,58 \\
\hline
\end{tabular}

Fuente: Autor (2021).

\subsection{DOSIS EXPERIMENTALES Y EVALUACIONES REALIZADAS}

Las dosis experimentales se realizaron en el laboratorio utilizando una hormigonera eléctrica estacionaria. Primero, el rasgo estándar se dosificó en la Tabla 3, y luego los otros rasgos con los respectivos niveles de sustitución adoptados, presentados en la Tabla 4.

Como principal criterio de evaluación, se adoptaron las posibles influencias de la introducción de cenizas de caldera en las dosis en relación con las propiedades: trabajabilidad del hormigón en estado fresco, densidad y resistencia a la compresión.

Para evaluar la influencia de la introducción de cenizas en la dosificación en relación con la trabajabilidad del concreto en estado fresco, el ensayo se realizó en todas las dosis para determinar la consistencia inicial por la reducción del tronco del cono, según NBR 16889 ABNT (2020). La consistencia inicial adoptada como estándar fue de entre $40 \mathrm{~mm}$ y $60 \mathrm{~mm}$. Después de alcanzar la consistencia inicial, el aditivo superplastificante se agregó a las dosis con el objetivo de aumentar la trabajabilidad

RC: 97179

Disponible: https://www.nucleodoconhecimento.com.br/ingenieria-civil/hormigonestructural 
y alcanzar la propagación entre 600 y $650 \mathrm{~mm}$, que se determinó en todas las dosis de acuerdo con NBR 15823-2 ABNT (2017).

En cuanto a la densidad, se determinó según NBR 9833 ABNT (2008).

La evaluación de la resistencia a la compresión se realizó a través de moldeos de muestras cilíndricas con dimensiones de 10x20mm, según NBR 5738 ABNT (2016). Dos muestras de cada serie se sometieron a la prueba para determinar la resistencia a la compresión axial a las edades de 7 días, 14 días y 28 días, según NBR 5739 ABNT (2018).

\subsection{RESULTADOS Y DEBATES}

En la dosificación estándar del rasgo, se observó que el agua predicha en el rasgo era suficiente para lograr la consistencia inicial, porque se obtuvo el índice de consistencia inicial de $52 \mathrm{~mm}$. Por lo tanto, se introdujo el aditivo superplastificante en la mezcla y se estableció el tiempo de mezcla de 8 minutos después de la dosificación del aditivo superplastificante. Este tiempo de mezcla es el tiempo medio determinado por el fabricante para contemplar el efecto completo del aditivo. Pasado el tiempo de mezcla, se determinó la propagación y se obtuvieron $620 \mathrm{~mm}$ como resultado, es decir, dentro de los parámetros previamente establecidos.

Después de la dosis traza estándar, el rastro se dosificó con un contenido de reemplazo del $10 \%$ del agregado muy delgado por cenizas de caldera. En esta dosificación, utilizando la misma cantidad de agua en relación con el rasgo estándar, se obtuvo un índice de consistencia inicial de $49 \mathrm{~mm}$. Se observó, por lo tanto, que la introducción de cenizas causó una ligera caída en la consistencia inicial del concreto. Sin embargo, estando aún dentro de los parámetros inicialmente establecidos, se dosificó el aditivo superplastificante y respetando el tiempo estándar de 8 minutos para la mezcla, posteriormente se determinó la propagación y se obtuvieron $600 \mathrm{~mm}$ de apertura.

RC: 97179

Disponible: https://www.nucleodoconhecimento.com.br/ingenieria-civil/hormigonestructural 
En secuencia, el rastro se dosificó con un contenido de reemplazo del $20 \%$ del agregado muy delgado por cenizas de caldera. Con la misma cantidad de agua utilizada en las dosis anteriores, se obtuvo el índice de consistencia inicial de $50 \mathrm{~mm}$. Con la dosificación del aditivo superplastificante y el final del tiempo de mezcla estándar, se extendió la apertura de $600 \mathrm{~mm}$, es decir, un comportamiento muy similar a la dosis anterior, con un contenido de reemplazo del $10 \%$ del agregado muy fino por cenizas de caldera.

Finalmente, la traza se dosificó con un contenido de reemplazo del $30 \%$ del agregado muy fino por cenizas de caldera. Manteniendo la cantidad de agua de las dosis anteriores, se obtuvo el índice de consistencia inicial de $38 \mathrm{~mm}$, es decir, por debajo del parámetro inicial establecido. En este caso, se observó que la introducción de cenizas en la mezcla interfería en la consistencia inicial como advirtió Neville (1997), pero se optó por la secuencia de dosificación porque el índice de consistencia inicial obtenido estaba muy cerca del límite inferior establecido, que era de $40 \mathrm{~mm}$. Después de la dosificación del aditivo superplastificante y el final del tiempo de mezcla, se extendieron $560 \mathrm{~mm}$. Por lo tanto, por debajo del parámetro inicialmente.

En el caso específico de la dosis con un contenido de reemplazo del $30 \%$ del agregado muy fino por cenizas de caldera, se decidió aumentar la dosis del aditivo superplastificante para lograr el mínimo reflejo predeterminado. Por lo tanto, la dosis del aditivo superplastificante se incrementó del $1 \%$ al $1,5 \%$ en el peso del cemento. Después de esta adición de aditivo la mezcla y respetado nuevamente el tiempo de mezcla para la acción total del aditivo, se obtuvo la propagación de $610 \mathrm{~mm}$, es decir, el ajuste de los parámetros preestablecidos.La trabajabilidad es una propiedad muy importante, porque "una mezcla de concreto que no se puede tirar fácilmente o densa en su totalidad no proporcionará las características de resistencia y durabilidad esperadas" (MEHTA; MONTEIRO, 1994).

RC: 97179

Disponible: https://www.nucleodoconhecimento.com.br/ingenieria-civil/hormigonestructural 
Las densidades encontradas según NBR 9833 ABNT (2018) se presentan en la Tabla 5.

Tabla 5 - Densidades determinadas

\begin{tabular}{|l|l|}
\hline $\begin{array}{l}\text { Identificación de la } \\
\text { serie }\end{array}$ & Densidad $\mathbf{( k g / \mathbf { m } ^ { 3 } )}$ \\
\hline Carrera estándar & 2335 \\
\hline Dash con $\mathbf{1 0} \%$ gris & 2078 \\
\hline Dash con $\mathbf{2 0 \%}$ de gris & 2070 \\
\hline Dash con $\mathbf{3 0 \%}$ de gris & 2066 \\
\hline
\end{tabular}

Fuente: Autor (2021).

Se observó que hubo una caída del $11 \%$ de densidad, comparando la traza estándar con la traza con el contenido de reemplazo del $10 \%$ del agregado muy fino por cenizas de caldera. Para los siguientes rasgos, con niveles de sustitución de $20 \%$ y $30 \%$, las disminuciones de densidad registradas fueron del orden de $0,4 \%$ y $0,2 \%$, respectivamente, comparando el rasgo analizado con el rasgo anterior, considerando el orden gradual y progresivo de los contenidos de reemplazo del agregado muy delgado por cenizas de caldera.

Los resultados encontrados en las pruebas para determinar la resistencia a la compresión axial se presentan en la Tabla 6.

Tabla 6 - Resistencia a la compresión (MPa) por serie

\begin{tabular}{|l|l|l|l|}
\hline $\begin{array}{l}\text { Resistencia a la serie/edad/compresión } \\
\text { (MPa) }\end{array}$ & $\mathbf{7}$ días & $\mathbf{1 4}$ días & $\mathbf{2 8}$ días \\
\hline Carrera estándar & 18,1 & 21,0 & 30,3 \\
\hline Dash con 10\% gris & 14,6 & 18,0 & 19,6 \\
\hline
\end{tabular}

RC: 97179

Disponible: https://www.nucleodoconhecimento.com.br/ingenieria-civil/hormigonestructural 


\begin{tabular}{|l|l|l|l|}
\hline Dash con $\mathbf{2 0} \%$ de gris & 15,2 & 18,3 & 20,2 \\
\hline Dash con $\mathbf{3 0} \%$ de gris & 14,6 & 18,5 & 19,8 \\
\hline
\end{tabular}

Fuente: Autor (2021).

Se verificó por los resultados obtenidos que las dosis con el uso de cenizas de caldera mostraron resistencias a la compresión más bajas que el rasgo estándar adoptado en todas las edades probadas. La caída media en la resistencia a la compresión de las trazas utilizando cenizas de caldera en relación con la carrera estándar se presentó como se muestra en la Tabla 7.

Tabla 7 - Porcentaje de caída de la resistencia a la compresión en relación con la carrera estándar

\begin{tabular}{|l|c|c|c|}
\hline Rasgo/Edad & $\mathbf{7}$ días & $\mathbf{1 4}$ días & $\mathbf{2 8}$ días \\
\hline $\begin{array}{l}\text { Dash con 10\% gris } \\
\text { Dash con 20\% de }\end{array}$ & $19 \%$ & $14 \%$ & $35 \%$ \\
\hline $\begin{array}{l}\text { gris } \\
\text { Dash con 30\% de } \\
\text { gris }\end{array}$ & $19 \%$ & $12 \%$ & $33 \%$ \\
\hline
\end{tabular}

Fuente: Autor (2021).

Se observó que en todos los rasgos con el uso de ceniza, el porcentaje de caída en la resistencia a la compresión en relación con el trazo estándar se comportó de manera similar independientemente del contenido de sustitución utilizado.

En cuanto al color de los hormigones dosificados, se encontró que tanto en estado fresco como endurecido, el contenido de reemplazo de agregados muy finos por cenizas de caldera influyó evidentemente en este aspecto visual, ya que debido a que el color de las cenizas de calderas era similar al del cemento Portland, los hormigones con mayores niveles de uso de cenizas se presentaban visualmente

RC: 97179

Disponible: https://www.nucleodoconhecimento.com.br/ingenieria-civil/hormigonestructural 
como hormigón con mayor consumo de cemento, es decir, con el color más oscuro mayor será el contenido de reemplazo adoptado.

\section{CONSIDERACIONES FINALES}

La ceniza de caldera industrial se clasificó como ligera y con un rango granulométrico ligeramente por debajo del rango muy delgado, ambas clasificaciones se aplican a agregados, por lo que se concluyó su aplicación como agregado ligero y muy delgado.

Con un aumento en el contenido del agregado reemplazado por cenizas de caldera industrial en la dosificación, hubo una influencia en la trabajabilidad del concreto, observada por la reducción de la reducción inicial y en la dispersión final. Por lo tanto, se concluyó la importancia de adoptar el método de reemplazo parcial del agregado para el uso de cenizas de caldera industrial, ya que se adoptó el reemplazo total del agregado, posiblemente habría un impacto aún mayor en esta propiedad del concreto en el estado fresco.

Se concluyó la importante contribución del aditivo superplastificante en las dosis con el uso de cenizas de calderas industriales, con el objetivo de compensar la pérdida de trabajabilidad causada por el uso de cenizas de calderas. Se entendió que con aditivos plastificificizantes, no sería posible lograr una trabajabilidad satisfactoria en los hormigones dosificados con la ceniza de la caldera industrial debido a la naturaleza de la ceniza y su gran superficie específica, lo que se traduce en un mayor consumo de agua en las dosis.

En cuanto a la densidad, se concluyó que con la sustitución del agregado por cenizas de caldera industrial, hubo una disminución de la densidad relacionada con el aumento en el contenido de reemplazo, es decir, cuanto mayor sea el contenido de reemplazo del agregado por la ceniza de caldera industrial, menor será la densidad verificada. Se consideró muy importante en futuras dosis observar la

RC: 97179

Disponible: https://www.nucleodoconhecimento.com.br/ingenieria-civil/hormigonestructural 
influencia causada por el uso de cenizas de caldera industrial en las dosis en relación con esta propiedad de hormigón.

El rendimiento de la resistencia a la compresión de los hormigones dosificados con cenizas de caldera industrial fue similar independientemente de las variaciones en el contenido de reemplazo del agregado. Sin embargo, hubo una disminución en esta propiedad en relación con el rasgo estándar, es decir, sin el uso del residuo. Por lo tanto, esta reducción de la resistencia debe considerarse en dosis futuras cuando se trata de dosificación a dosis en comparación sin el uso de residuos.

Se concluyó, por lo tanto, que el uso de cenizas de caldera industrial en la medición de hormigón estructural es factible, porque se lograron características satisfactorias en relación con la trabajabilidad y el rendimiento de la resistencia a la compresión de acuerdo con los parámetros normativos. Por lo tanto, a través de este estudio, fue posible identificar comportamientos iniciales del hormigón dosificado con cenizas de caldera y demostrar la viabilidad de utilizar este residuo.

Se entiende que será necesario continuar la investigación para mejoras, asegurando el buen rendimiento del hormigón en el estado fresco y endurecido, así como durante la vida útil de los edificios.

\section{REFERENCIAS}

Associação brasileira de normas técnicas. NBR 11579: Cimento Portland Determinação do índice de finura por meio da peneira 75 um ( $n^{0}$ 200). Rio de Janeiro, 2012.

Associação brasileira de normas técnicas. NBR 15823-2: Concreto autoadensável: Parte 2 - Determinação do espalhamento, do tempo de escoamento e do índice de estabilidade visual - Método do cone de Abrams. Rio de Janeiro, 2017.

$\mathrm{RC}: 97179$

Disponible: https://www.nucleodoconhecimento.com.br/ingenieria-civil/hormigonestructural 
Associação brasileira de normas técnicas. NBR 16889: Concreto - determinação da consistência pelo abatimento do tronco de cone. Rio de Janeiro, 2020.

Associação brasileira de normas técnicas. NBR 5738: Concreto - procedimento para moldagem e cura de corpos de prova. Rio de Janeiro, 2016.

Associação brasileira de normas técnicas. NBR 5739: Concreto - ensaio de compressão em corpos de prova cilíndricos. Rio de Janeiro, 2018.

Associação brasileira de normas técnicas. NBR NM 248: Agregados - Determinação da composição granulométrica. Rio de Janeiro, 2003.

Associação brasileira de normas técnicas. NM 52: Agregados - Determinação da massa unitária e volume de vazios. Rio de Janeiro, 2009.

Associação brasileira de normas técnicas. NBR 9833: Concreto fresco Determinação da massa específica, do rendimento e do teor de ar pelo método gravimétrico. Rio de Janeiro, 2008.

Bauer, Luiz Alfredo Falcao. Materiais de construção. Rio de Janeiro: LTC, 2008.

Cavalcante, Zedequias Vieira. A Importância da Revolução Industrial no Mundo da tecnologia. Unicesumar. Maringá, 2011. Encontro Internacional de Produção Científica. Disponível em: https://www.unicesumar.edu.br/epcc-2011/wpcontent/uploads/sites/86/2016/07/zedequias_vieira_cavalcante2.pdf. Acesso em: 10 abr. 2021.

Ganzala, Gabryelly Godois. A Industrialização, impactos ambientais e a necessidade de desenvolvimento de políticas ambientais sustentáveis no século XXI. 2018. Centro Universitário Internacional - UNINTER. Disponível em: https://repositorio.uninter.com/bitstream/handle/1/295/1355104\%20-

$\mathrm{RC}: 97179$

Disponible: https://www.nucleodoconhecimento.com.br/ingenieria-civil/hormigonestructural 
\%20GABRYELLY\%20GODOIS\%20GANZALA.pdf?sequence=1\&isAllowed=y. Acesso em: 4 abr. 2021.

MEHTA, P. K. e MONTEIRO, P. J. M. Concreto: Estrutura, Propriedades e Materiais. São Paulo: Editora Pini, 1994.

Neville, Adam Matthew. Propriedades do concreto. São Paulo: Editora Pini, 1997.

Enviado: Agosto de 2021.

Agosto: Septiembre, 2021.

RC: 97179

Disponible: https://www.nucleodoconhecimento.com.br/ingenieria-civil/hormigonestructural 